\title{
A Novel Adaptive Neural Network Compensator as Applied to Position Control of a Pneumatic System
}

\author{
Behrad Dehghan ${ }^{1}$, Sasan Taghizadeh ${ }^{2}$, Brian Surgenor ${ }^{1}$, Mohammed Abu-Mallouh ${ }^{3}$ \\ ${ }^{1}$ Department of Mechanical and Materials Engineering, Queen's University, Kingston, Canada \\ ${ }^{2}$ Department of Electrical and Computer Engineering, University of Waterloo, Waterloo, Canada \\ ${ }^{3}$ Departmnet of Mechatronics Engineering, Hashemite University, Zarqa, Jordan \\ E-mail:behrad.dehghan@queensu.ca,staghiza@uwaterloo.ca,surgenor@me.queensu.ca,mmallouh@hu.edu.jo \\ Received August 13, 2011; revised September 5, 2011; accepted September 25, 2011
}

\begin{abstract}
Considerable research has been conducted on the control of pneumatic systems. However, nonlinearities continue to limit their performance. To compensate, advanced nonlinear and adaptive control strategies can be used. But the more successful advanced strategies typically need a mathematical model of the system to be controlled. The advantage of neural networks is that they do not require a model. This paper reports on a study whose objective is to explore the potential of a novel adaptive on-line neural network compensator (ANNC) for the position control of a pneumatic gantry robot. It was found that by combining ANNC with a traditional PID controller, tracking performance could be improved on the order of $45 \%$ to $70 \%$. This level of performance was achieved after careful tuning of both the ANNC and PID components. The paper sets out to document the ANNC algorithm, the adopted tuning procedure, and presents experimental results that illustrate the adaptive nature of $\mathrm{NN}$ and confirms the performance achievable with ANNC. A major contribution is demonstration that tuning of ANNC requires no more effort than the tuning of PID.
\end{abstract}

Keywords: Gantry Robot, Servopneumatics, Neural Networks, Adaptive Control, PID Control

\section{Introduction}

A large body of research is devoted to improving the performance of servo pneumatic systems. For example, a good comparative study of position tracking algorithms is given by Bone and Ning who used a sliding-mode controller [1]. As a more recent example, Wang et al compared three sliding mode schemes in a sinusoidal tracking application [2]. As an example of an intelligent (neuro fuzzy) controller in servo pneumatics, Gi et al. reported success with feedback linearization by means of a neural network (NN) toolbox [3]. The NN was trained off-line. Experimental results showed that the NN improved tracking performance relative to a non-compensated controller for a range of reference signal frequencies and amplitudes. The NN approach is particularly attractive as it does not need a model of the process, be it linear or nonlinear. The more successful advanced nonlinear and adaptive control strategies typically need a mathematical model of the system to be controlled [3-7].

Gross and Rattan in [4] conducted research on using $\mathrm{NN}$ as a compensator for velocity control of a pneumatic actuator. Simulation results were presented. Although they were satisfied by the results, there was no comparison against the performance of a conventional controller. In the context of pneumatic servo control, a NN was used in [5] to compensate for the time delay and nonlinear friction effects on a 2-link pneumatic manipulator. Simulation and experimental results were presented. The NN was trained on-line. However, there was no direct comparison of the simulation results with the experimental results. Standalone results in which the length of the connecting lines were varied showed good performance for time delays that ranged from 0.012 to $0.12 \mathrm{sec}$. As this is the level of delay experienced with the application in this paper, it further confirms the potential for a $\mathrm{NN}$ approach to the problem at hand.

Wang and Peng [6] used an on-line NN as a model predictor for position control of a pneumatic actuator with proportional pressure valves. Only simulation results were presented. Although they concluded that the $\mathrm{NN}$ had a significant positive impact on performance, they did not benchmark their results with other controllers. Kothapalli and Hassan [7] tried to use an off-line 
$\mathrm{NN}$ to adjust the gains of a PI position controller of a pneumatic system. Again, only simulation results were presented. They showed that the NN could reduce overshoot, rise time and the steady-state error of a step response. The effect of adding pay-load was discussed but not quantified.

It should be pointed out that only in the cases of [1] and [3] were experimental results benchmarked against conventional PID control. In those papers that employed NN's it should also be observed that no details were given on the tuning of the NN. In an earlier paper, a novel adaptive neural network compensator (ANNC) was applied to a contour tracking application with a pneumatic gantry robot [8]. The results were disappointing, with only a $20 \%$ improvement in tracking performance as benchmarked against PID. The conclusion at the time was that this degree of improvement with ANNC did not warrant the extra effort required for tuning and implementation. It was subsequently determined that both the hardware and controller configurations were less than optimal, and with proper tuning, truly significant performance gains could be achieved. The paper sets out to document the ANNC algorithm, the adopted tuning procedure, and presents experimental results that illustrate the adaptive nature of NN and confirms the level of performance achievable with ANNC.

\section{Pneumatic System and Controller}

The apparatus under test is a pneumatic gantry robot, as illustrated in Figure 1. Technically, the robot has 3 degrees of freedom. The $\mathrm{y}$-axis consists of two rodless pneumatic cylinders that form the sides of the gantry. The $\mathrm{x}$-axis is a single rodless pneumatic cylinder (bore $32 \mathrm{~mm}$, stroke $1 \mathrm{~m}$ ). The $\mathrm{x}$-axis cylinder acts as the bridge between the two y-axis cylinders. The z-axis is in the same direction as the $y$-axis, but is available as the third degree of freedom. However, for the reported experiments in this paper only the $\mathrm{x}$-axis cylinder was tested.

The cylinder is controlled by a 5 port 3 way proportional flow valve. Position and velocity were measured directly with wire linked potentiometers and tachometers, respectively. The wire linkage uses a constant torsion spring and this "wire force" cannot be neglected. Pressure transducers measure the differential air pressure directly across a cylinder. Data acquisition and control was PC-based with a dSPACE®/DSP as the data acquisition hardware/software and MATLAB/Simulink ${ }^{\circledR}$ as the control software. Sampling time was $1 \mathrm{msec}$. The pressure supply was $500 \mathrm{kPa}$ (72.5 psi) as regulated by a manual pressure regulator. The weight of $\mathrm{x}$-axis slide is $7.3 \mathrm{~kg}$. The Coulomb friction for the $\mathrm{x}$-axis is calculated.

In Figure 2 the controller and the pneumatic circuit

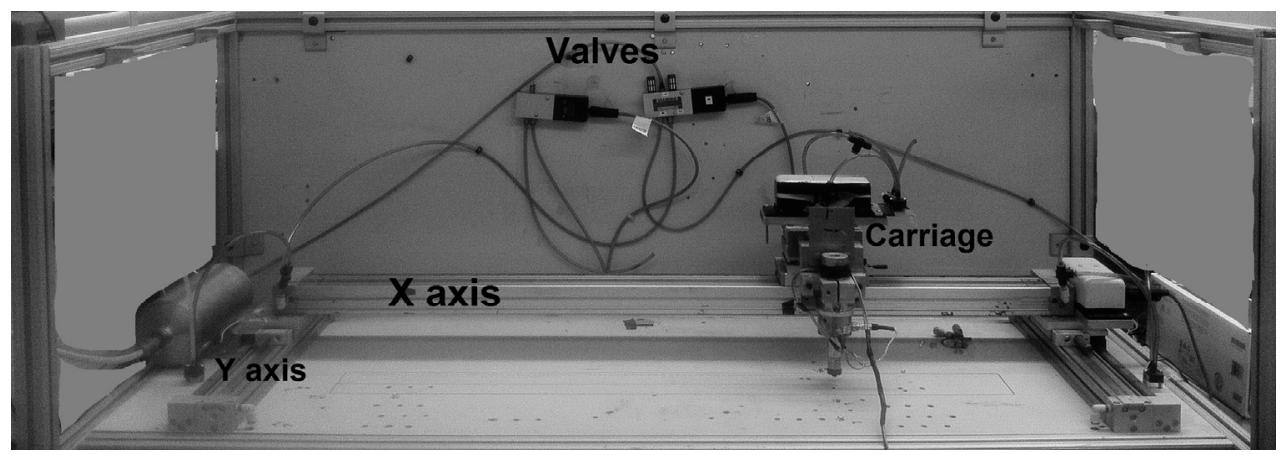

Figure 1. Pneumatic gantry robot with $x$-axis labelled.

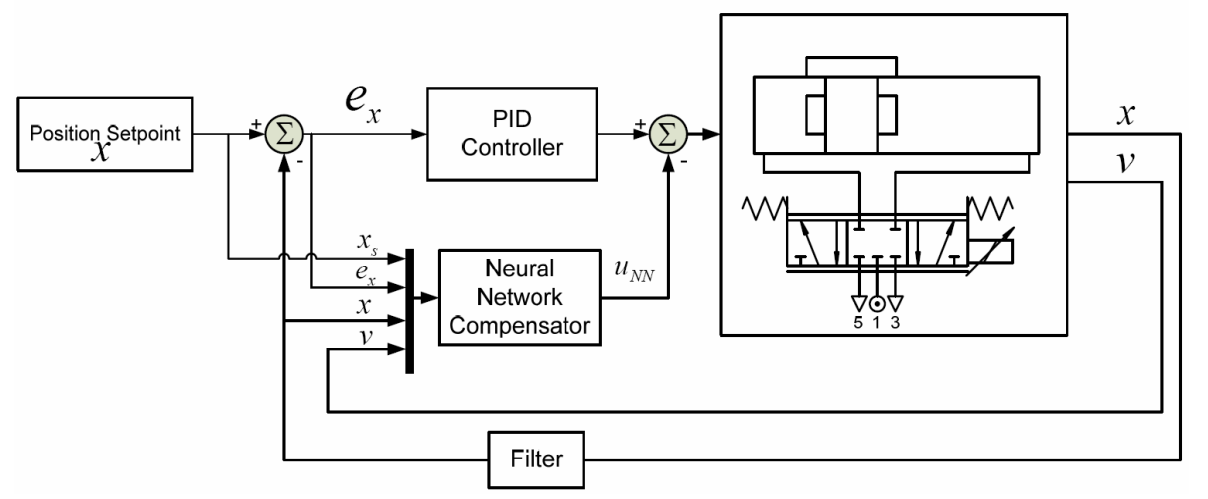

Figure 2. Pneumatic circuit with PID controller and ANN compensator. 
under test is illustrated. The controller is a fixed gain PID that is tuned with a $0.33 \mathrm{~Hz}$ sinusoidal reference signal:

$$
u_{\mathrm{PID}}=K_{p} e_{x}+K_{i} \int e_{x} \mathrm{~d} t+K_{d} \frac{\mathrm{d} e_{x}}{\mathrm{~d} t}
$$

The neural network output $u_{N N}$ is subtracted from the PID output to give as the control signal:

$$
u=u_{\mathrm{PID}}-u_{\mathrm{NN}}
$$

The neural network signal is subtracted from the PID output and the difference provides the input signal to the control valve. A first order filter is used on the position signal.

\section{Adaptive Neural Network Compensator}

The algorithm for the Adaptive Neural Network Compensator (ANNC) is based upon the Modified Back Propagation Method (MBPM) originally proposed by Lewis [9]. The original MBPM was adapted to real time control applications by Campa et al. [10]. He provided a Simulink ${ }^{\circledR}$ block in MATLAB ${ }^{\circledR}$ which models the MPBM of Lewis. Taghizadeh et al. [8] in turn took the simulation model of Campa and adapted it for the experimental application seen in this paper.

In practice, ANNC provides a feed-forward signal that linearizes the system to enable application of a linear controller to a nonlinear system. The key adaptive parameters are the weights. It is assumed that for every smooth function $f(x)$, there exists a NN such that:

$$
f(x)=W^{T} \sigma\left(V^{T} x\right)+\varepsilon
$$

where $W$ is the weight vector for the output layer, $V$ is the weight vector for the hidden layer and $\varepsilon$ is the difference between $f(x)$ and the NN. It also noted that, in the presence of unmodeled disturbances, the tracking error does not vanish but it is bounded. Furthermore, relatively small tracking errors can be achieved with relatively high NN gains. The only drawback is that in the training phase, slow learning rates can cause the $\mathrm{NN}$ to oscillate over the local minimum. The advantage of this structure is that the weights can be easily initialized and tuned online. No off-line training is required. Lewis et al. [11] demonstrated the viability of the original technique. But they did not address key structural issues such as the effect of the number of nodes and provided no experimental results.

\subsection{ANNC Algorithm}

As illustrated in Figure 3, the basic structure for the ANNC is that of a three layer neural network. The NN has to be optimized in terms of the number of nodes in both the input and hidden layers. A key design parameter

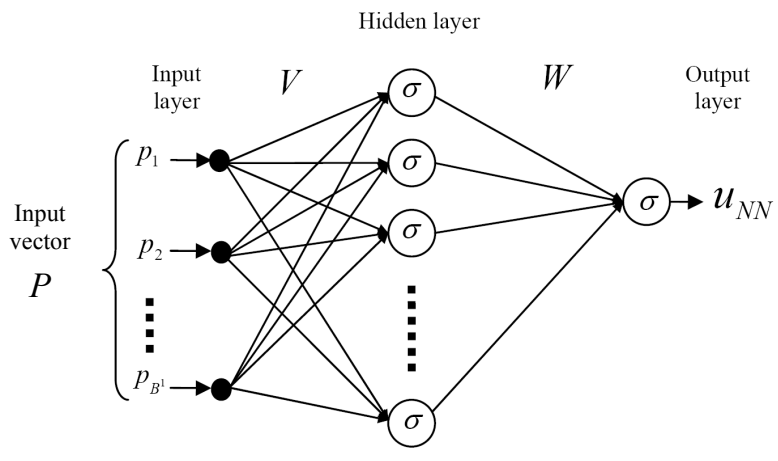

Figure 3. Three layer ANN with one output.

is the nature of the activation functions for each node. For sigmoidal NN's, the activation function $\sigma_{i}^{L}$ for node $i$ in layer $L$ is commonly given as:

$$
\sigma_{i}^{L}=\frac{1}{1+e^{-n e t_{i}^{L}}}, i=1,2, \cdots, B^{L}
$$

where $B^{L}$ is the number of nodes in layer $L$ and $L=1,2$ and 3. The function $n e t_{i}^{L}$ is the sum of the inputs to node $i$ in layer $L$ and is defined for the hidden layer ( $L=$ 2) and output layer $(L=3)$ as follows:

$$
\begin{gathered}
n e t_{i}^{2}=\sum_{j=1}^{B^{1}} V_{i, j} p_{j}+b_{i}^{2}, i=1,2, \cdots, B^{2} \\
n e t_{1}^{3}=\sum_{j=1}^{B^{2}} W_{1, j} a_{j}^{2}+b_{1}^{3}
\end{gathered}
$$

where $V_{i, j}$ is the weight connecting node $i$ in the hidden layer $(L=2)$ and input $p_{j}$ of the input layer, $p_{j}$ is $j^{\text {th }}$ input of the input layer, $W_{1, j}$ is the weight connecting the output node in the output layer and the output of node $j$ in the hidden layer $\left(a_{j}^{2}\right), B^{2}$ is number of nodes in the hidden layer, $b_{i}^{2}$ and $b_{1}^{3}$ are the bias of node $i$ in the hidden and output layer, respectively.

The standard sigmoid activation function given as Equation (4) is used as the basis for the ANNC. For back-propagation based NN's, the updates to tuning weights are usually given as:

$$
\begin{gathered}
\dot{W}=F \sigma\left(V^{T} P\right) e \\
\dot{V}=G P\left(W^{T} \dot{\sigma}\left(V^{T} P\right) e\right)^{T}
\end{gathered}
$$

where $W$ is the weight vector for the output layer, $V$ is the weight vector for the hidden layer, $F$ is the learning rate for $W$ and $G$ is the learning rate for $V, P$ is the input vector and $\mathrm{e}$ is the error in the input. The training algorithm for the weights is given as:

$$
\begin{gathered}
\left.\dot{W}=F \sigma\left(V^{T} P\right) e-F \dot{\sigma}\left(V^{T} P\right) V^{T} P e-\lambda F W\|e\|\right] \\
\dot{V}=G P\left(W^{T} \dot{\sigma}\left(V^{T} P\right) e\right)^{T}-\lambda G V\|e\|
\end{gathered}
$$


where $\lambda$ is a small positive tunable parameter whose function is to help deal with unmodeled dynamics [9]. Examination of Equations (9) and (10) reveal that they consist of a standard back-propagation term $\left(1^{\text {st }}\right.$ term in equations), plus the error modification term taken from adaptive control (last term), plus a novel second-order forward-propagation term taken from the backpropagation network ( $2^{\text {nd }}$ term in Equation (9)).

In the ANNC the neural network output $u_{\mathrm{NN}}$ is given as:

$$
u_{\mathrm{NN}}=\sigma_{1}^{3}\left(\sum_{j=1}^{B^{2}}\left(W_{1, j} a_{j}^{2}\right)+b_{1}^{3}+D\right)
$$

where an additional parameter $D$ has been added to the standard NN output to overcome higher order modeling errors. The parameter $D$ is given by:

$$
D=-k_{z}(\|Z\|+\bar{Z}) e-k_{v} e
$$

where $\bar{Z}$ is the maximum expected value of $Z, k_{z}$ and $k_{v}$ are gain terms and the matrix $Z$ is given by:

$$
Z=\left[\begin{array}{cc}
W & 0 \\
0 & V
\end{array}\right]
$$

The advantage of the ANNC is that it is designed expressly for on-line training. Thus, the weights can be easily initialized and tuned on-line. No off-line training is required. The ANNC tuning algorithm makes the NN strictly state passive. This means that bounded weights are guaranteed for all applications, even in the presence of unmodeled disturbances and dynamics.

\subsection{ANNC Implementation and Tuning}

For the implementation of ANNC, the first question is the number of inputs. The selection of inputs is a key determinant of performance. There are 3 concerns which must be considered:

Inter-dependency of variables: Two or more interdependent variables may carry significant information that a subset would not. Thus, variables cannot be independently selected.

Curse of dimensionality: The addition of an input node to a network adds a dimension to the space and the number of weights increases exponentially. The performance of a network can be improved by eliminating unnecessary inputs. But equally so, performance depends upon having an adequate number of necessary inputs. Unfortunately, there is no rigorous method of identifying which are "unnecessary" and which are "necessary".

Redundancy of variables: Different inputs may carry the same information, because they are correlated. A subset of uncorrelated inputs can have superior perform- ance relative to a full set of correlated and uncorrelated inputs.

One approach is to use a combination of problem domain knowledge and standard statistical tests to select inputs. A second approach is to experimentally add and remove combinations of inputs, building a new network each time and testing the result. A third approach is to conduct a Sensitivity Analysis to rates the importance of variables with respect to a particular model. For this study, the second approach was used.

After input selection and setting up the network (with zero as initial values for the weights $V$ and $W$ ), the next step is tuning of the ANNC parameters. Figure 4 illustrates the procedure used to tune the ANNC as developed for this study. Table 1 gives the tuned result (as taken from [8]). Again, this set of parameters was obtained by trial and error, observing system performance when tracking a $0.33 \mathrm{~Hz}$ sinusoidal reference signal. Note that the 12 inputs indicated in Table $\mathbf{1}\left(n_{i}=12\right)$ are in fact a subset of the 4 inputs shown in Figure 2.

The first step involves initialization of the structural parameters. Given that for this application there is only

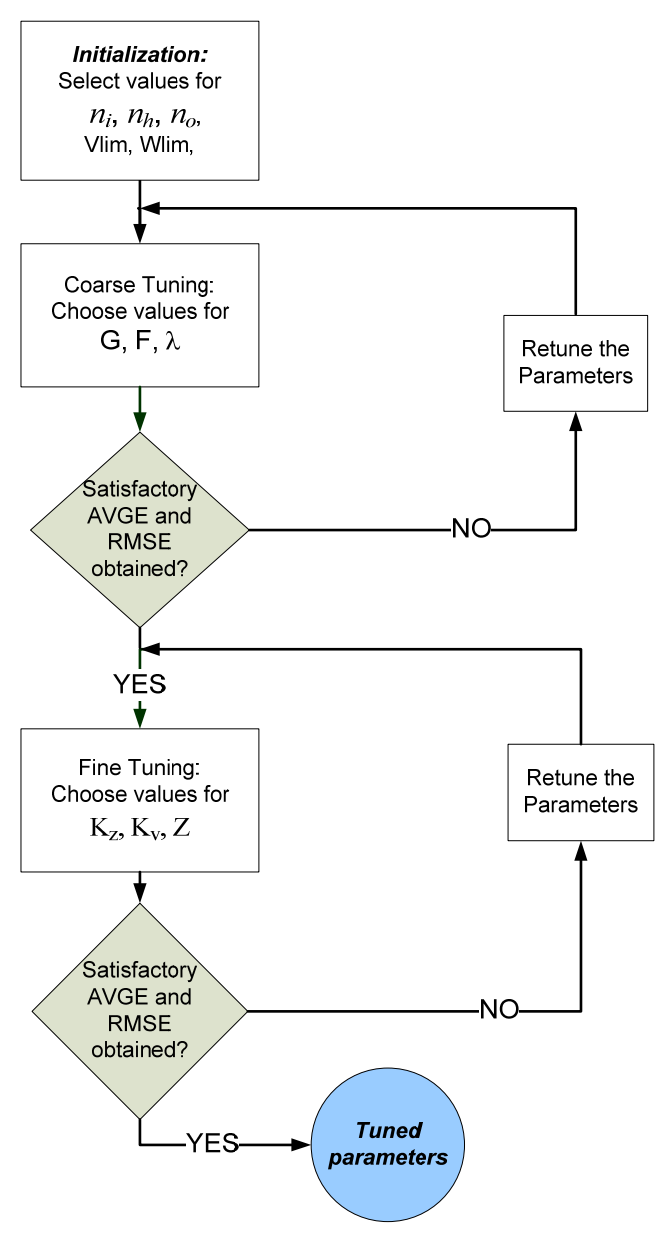

Figure 4. Flowchart for ANNC tuning procedure. 
Table 1. Tuned values of ANNC parameters.

\begin{tabular}{ccc}
\hline Parameter & Definition & Value \\
\hline$n_{i}$ & number of inputs & 12 \\
$n_{h}$ & number of nodes in hidden & 10 \\
$n_{o}$ & layer & \\
$G$ & number of outputs & 1 \\
$F$ & learning rate of $V$ & 1 \\
$\lambda$ & learning rate of $W$ & 1 \\
$S$ & adaption parameter & 1.5 \\
bias & slope of sigmoid activation & 1 \\
Lim $V$ & function & 1 \\
Lim $W$ & activation function bias & 10 \\
$K_{z}$ & limit of $V$ & 10 \\
$K_{v}$ & limit of $W$ & 0.5 \\
$Z$ & tuning parameter & 0.5 \\
\hline
\end{tabular}

one output, $n_{o}$ is set to 1 . Furthermore, bias can be set to 1 as an initial value as this is one of the adapting parameters. Finally, the weights have upper and lower limits. It was found that only variations in the upper limits had an affect and that values of 10 worked for all applications to date.

The second iteration involves tuning of the values for $G, F$ and $\lambda$. Large values of $G$ and $F$ enables a large value for $\lambda$. Thus, for this application, $G$ and $F$ were set to the same value $G F$. In practice, $G F$ and $\lambda$ are for the coarse tuning (which means that large changes in their values result in small changes to performance). $G, F$ and $\lambda$ are considered tuned when variation in performance is less than $\pm 10 \%$.

The third iteration involves tuning of the values for $K_{z}, K_{v}$ and $\bar{Z}$. It was found that they could be treated equally. Thus, for this application, $K_{z}, K_{v}$ and $\bar{Z}$ were set to the same value $K K Z$. In practice, $K K Z$ is for fine tuning (which means small changes in its value results in large changes to performance). $K_{z}, K_{v}$ and $\bar{Z}$ are considered tuned when variation in performance is less than $\pm 1 \%$.

In conclusion, one notes that there are 13 parameters in Table 1. If all 13 parameters had to be actively tuned, this would be seen as a significant practical drawback to the application of ANNC. However, the adopted tuning procedure highlights that in fact only 3 parameters require active tuning: $G F, \lambda$ and $K K Z$. With only 3 parameters to tune, this puts ANNC on the same footing as PID when it comes to tuning.

\section{Experimental Results}

As performance measures for the experimental results, the average absolute error $(A V G E)$ and Root mean square error $(R M S E)$ are calculated:

$$
\begin{aligned}
A V G E & =\frac{\sum_{1}^{N}(\mid \text { error } \mid)}{N} \\
R M S E & =\sqrt{\frac{\sum_{1}^{N}(\text { error })^{2}}{N}}
\end{aligned}
$$

Also, the percentage improvement in tracking performance for each case is calculated by:

$$
\begin{aligned}
& \% \triangle A V G E=\frac{A V G E-A V G E_{r e f}}{A V G E_{r e f}} \times 100 \\
& \% \Delta R M S E=\frac{R M S E-R M S E_{r e f}}{R M S E_{r e f}} \times 100
\end{aligned}
$$

\subsection{Sinusoidal Tracking Results}

Performance was measured as the system tracked a sinusoidal reference signal. Figure 5 illustrates a typical response. Note that the $15 \mathrm{~s}$ time frame is the window over which RMSE and $A V G E$ were calculated for all tests. Experiments were conducted with a regulated supply pressure of $500 \mathrm{kPa}$. In all cases the PID gains were $K_{p}=$ $2.25, K_{i}=9$ and $K_{d}=0.6$. These PID gains were obtained following the "half gain rule" used in industrial selftuning controllers [12].

Table 2 shows what happens if one deviates from the tuned values of Table 1. Thus, Table 2 illustrates the relative importance of the ANNC parameters. A negative sign for $\triangle R M S E$ and $\triangle A V G E$ means performance has improved relative to the reference case. Experiments were conducted with a $0.33 \mathrm{~Hz}$ sine wave input. The first line in Table 2 is the reference case which shows $A V$ $G E_{\text {ref }}=31.0$ and $R M S E_{\text {ref }}=34.8$ (as calculated with Equations (16) and (17), respectively). Subsequent lines illustrate the change in $A V G E$ and $R M S E$ relative to the reference line for a given parameter change.

The following observations can be made about Table 2:

- Changing learning rates $F, G$ does not significantly affect the result;

Table 2. Effect of changing ANNC parameters.

\begin{tabular}{ccccc}
\hline Parameter & $R M S E$ & $A V G E$ & $\triangle R M S E \%$ & $\triangle A V G E \%$ \\
\hline with ANNC & 34.8 & 31.0 & 0 & 0 \\
without ANNC & 51.7 & 45.9 & 49 & 44 \\
$F=0.05$ & 37.8 & 33.0 & 11 & 3.9 \\
$G=0.05$ & 35.2 & 30.8 & 3.6 & -3.0 \\
$G=0.05, \lambda=5$ & 42.6 & 38.9 & 34 & 22 \\
$\lambda=5$ & 49.5 & 50.5 & 45.5 & 59 \\
slope $=5$ & 69.7 & 61.7 & 105 & 94 \\
bias $=1$ & 34.7 & 31.2 & 2.1 & -1.6 \\
$K_{v}=0$ & 54.2 & 49.3 & 55 & 59 \\
$K_{v}=0, Z=0$ & 61.6 & 53.5 & 77 & 72 \\
\hline
\end{tabular}


- Increasing slope significantly degrades performance;

- Changing bias does not significantly affect the result

- Increasing adaptation parameter $\lambda$ significantly degrades performance;

- Changing the parameters $K_{v}$ and $Z$ significantly degrades performance.

The key takeaway from Table 2 is that there was no instance of performance significantly improving. This provides further evidence that the parameters in Table 1 are indeed "tuned".

Table 3 compares the performance of PID only and PID + ANNC for three tracking frequencies. The improvement with PID + ANNC relative to PID only is given by $\triangle R M S E$ and $\triangle A V G E$. One sees that PID +
ANNC is able to reduce the average error by $45 \%$ to $70 \%$ relative to PID only. Figures 5 and $\mathbf{6}$ provide the $0.1 \mathrm{~Hz}$ results for PID only and PID + ANNC, respectively. At a glance, the two figures look very similar. But an examination of the error trace shows that its amplitude in Figure 6 is less than its amplitude in Figure 5, which equates numerically to a $45 \%$ reduction in error. One should also note that the control signal trace in Figure 6 dampens to near steady state at the end of each $5 \mathrm{~s}$ cycle.

This result is comparable to Gi et al. [3] who reported a $74 \%$ improvement over PID. At the same time, recall that the NN of Gi et al. was off-line and had to be trained for each operating condition. The inherent advantage of ANNC is that it is an on-line NN and consequently trains
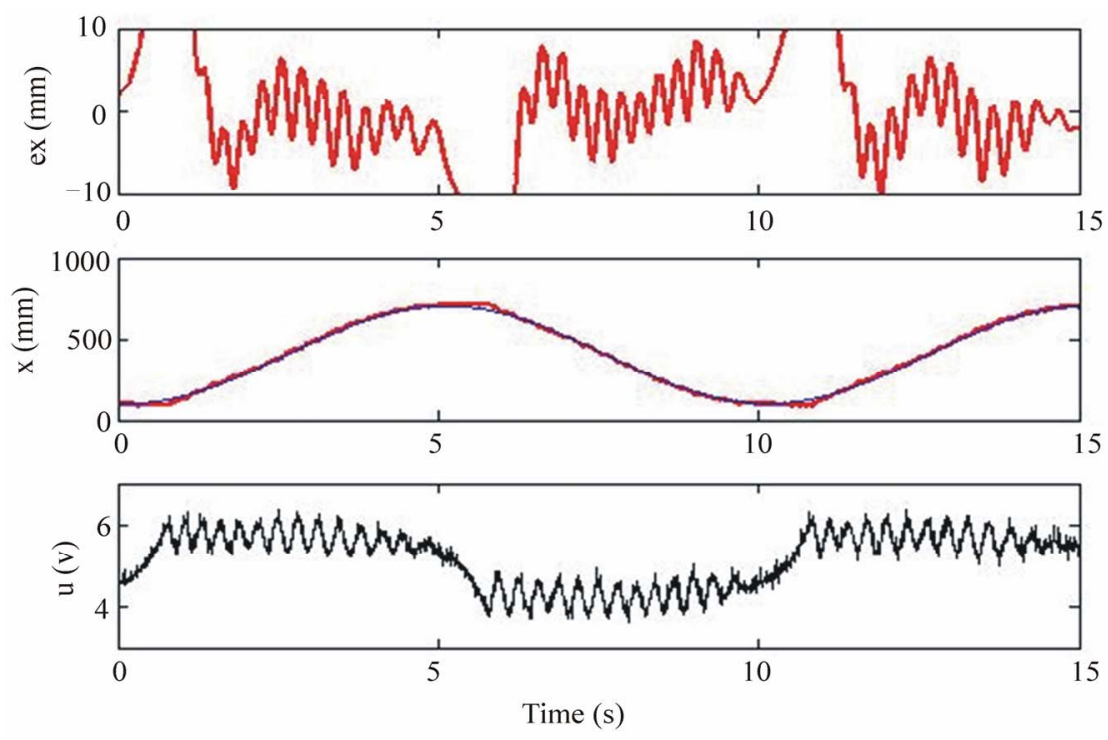

Figure 5. PID only response at $0.1 \mathrm{~Hz}(R M S E=13.67, A V G E=11.29)$.
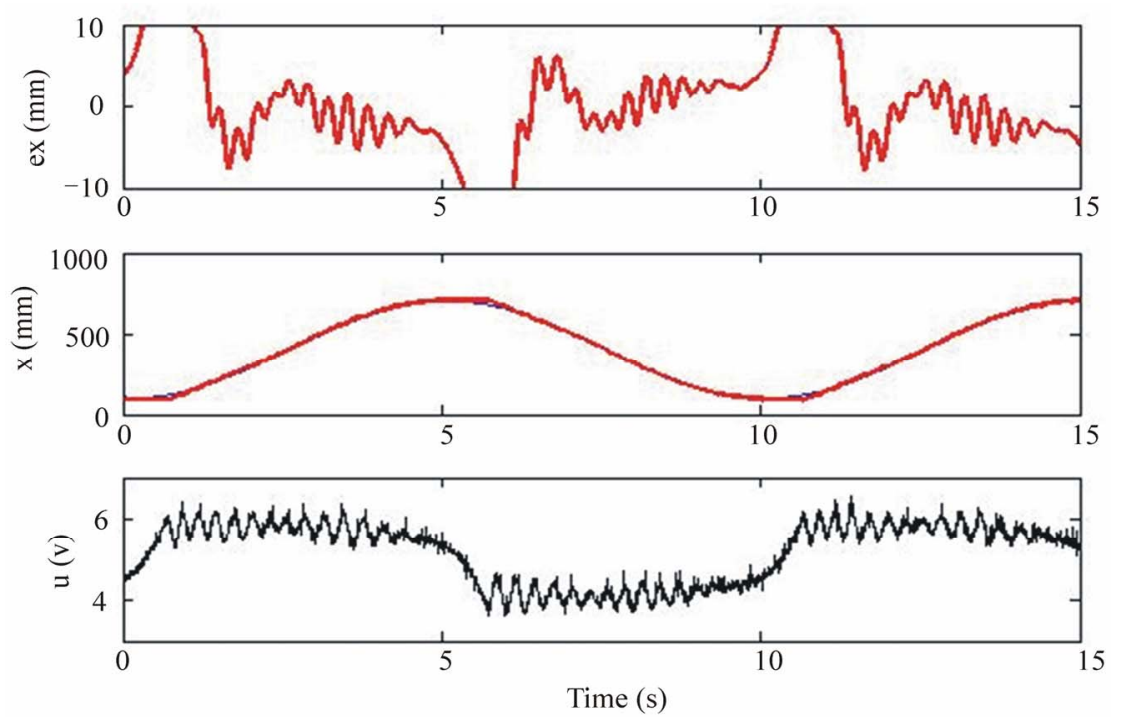

Figure 6. PID + ANNC response at $0.1 \mathrm{~Hz}(\mathrm{RMSE}=7.93, A V G E=6.16)$. 
Table 3. Improvement in performance with ANNC.

\begin{tabular}{ccccccc}
\hline & \multicolumn{2}{c}{ PID Only } & \multicolumn{2}{c}{ PID + ANNC } & \multicolumn{2}{c}{ Percent Change } \\
\hline Frequency & $R M S E$ & $A V G E$ & $R M S E$ & $A V G E$ & $\% \Delta R M S E$ & $\% \Delta A V G E$ \\
$0.1 \mathrm{~Hz}$ & 13.67 & 11.29 & 7.93 & 6.16 & -72 & -45 \\
$0.2 \mathrm{~Hz}$ & 19.92 & 18.13 & 9.75 & 8.37 & -51 & -53 \\
$0.5 \mathrm{~Hz}$ & 219.2 & 198.0 & 67.5 & 58.55 & -69 & -70 \\
\hline
\end{tabular}
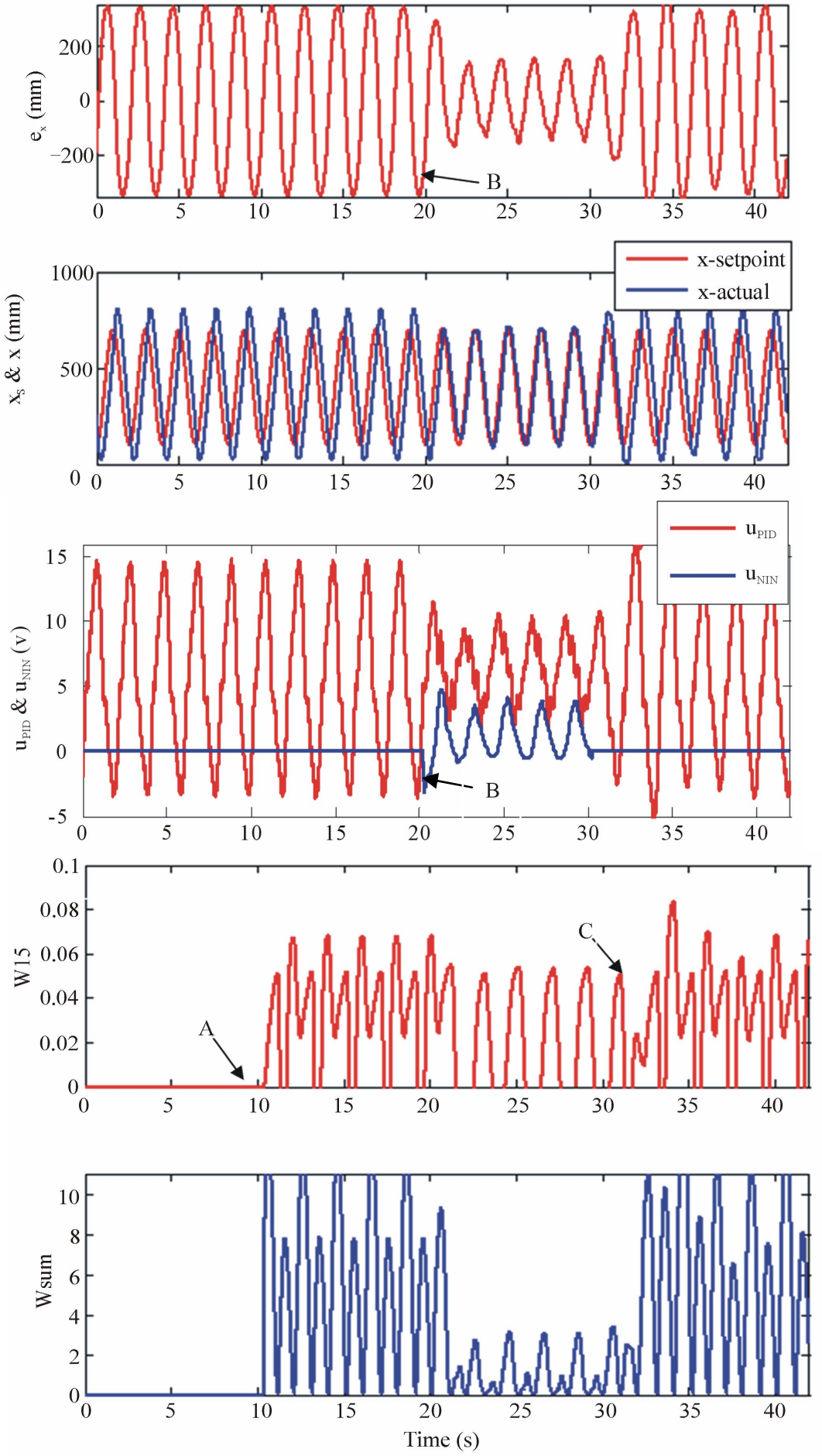

Figure 7. Transient behaviour of ANNC at $0.33 \mathrm{~Hz}$ with events A, B and C identified. 
itself as the operating condition changes. Finally, although accuracy is on the order of $\pm 6 \mathrm{~mm}$ which seems poor, as a percentage of stroke this equates to $\pm 0.6 \%$, which is comparable to the percent accuracy reported by [1] and [3].

\subsection{Transient Behaviour of ANNC}

A series of additional tests were conducted at $0.33 \mathrm{~Hz}$ to study the transient behaviour of ANNC and to confirm that it was indeed "adapting". Figure 7 illustrates system response when the ANNC is turned on and off. There are three events to highlight:

Event A $(10 \mathrm{~s})$ - ANNC learning starts $\left(u=u_{\mathrm{PID}}\right)$

Event B $(20 \mathrm{~s})$ - ANNC connected $\left(u=u_{\mathrm{PID}}-u_{\mathrm{NN}}\right)$

Event C $(30 \mathrm{~s})$ - ANNC disconnected $\left(u=u_{\mathrm{PID}}\right)$

The active adaptive nature of ANNC is clearly visible and the result for this particular case is a $71 \%$ reduction in $A V G E$. The weights reach their new values in just one cycle. Note that $W_{\text {sum }}$ is the sum of the elements of $W$ and thus exceeds the individual limit of 10 that seen in Table 1.

\section{Conclusions}

The potential of a novel adaptive on-line neural network compensator (ANNC) for the position control of a pneumatic gantry robot has been demonstrated. It was found that by combining ANNC with a traditional PID controller, tracking performance could be improved on the order of $45 \%$ to $70 \%$. This level of performance was achieved after careful tuning of both the ANNC and PID components. This paper documented the ANNC algorithm and its tuning procedure, and presented experimental results that illustrated the adaptive nature of $\mathrm{NN}$ and confirmed the performance achievable with ANNC. A major contribution is demonstration that tuning of ANNC requires no more effort than the tuning of PID, in that they both require the user to find values for only 3 parameters.

\section{References}

[1] G. M. Bone and S. Ning, "Experimental Comparison of Position Tracking Control Algorithms for Pneumatic Cylinder Actuators," IEEE/ASME Transactions on Mechatronics, Vol. 12, No. 5, 2007, pp. 557-561.

\section{doi:10.1109/TMECH.2007.905718}

[2] Y. Wang, H. Su, K. Harrington and G. Fischer, "Sliding Mode Control of Piezoelectric Valve Regulated Pneumatic Actuator for MRI-Compatible Robotic Intervention," Proceeding of ASME Dynamic Systems and Control Conference, Cambridge, 12-15 September 2010, pp. 1-6.

[3] S. C. Gi, K. L. Han and H. C. Gi, "A Study on Tracking Position Control of Pneumatic Actuators using Neural Network," Proceeding of 24th Annual Conference on IEEE Industrial Electronics Society, Aachen, 31 Augudt-4 September 1998, pp. 1749-1753.

[4] D. C. Gross and K.S. Rattan, "Pneumatic Cylinder Trajectory Tracking Control using a Feedforward Multilayer Neural Network," Proceedings of the IEEE $1997 \mathrm{Na}$ tional Aerospace and Electronics Conference, Dayton, 14-17 July 1997, pp. 777-784. doi:10.1109/NAECON.1997.622728

[5] Y. Li and T. Asakura, "A Study on Neural Network Control of Explosion-Proof 2-link Pneumatic Manipulator," 2003 IEEE/ASME International Conference on Advanced Intelligent Mechatronics, Kobe, 20-24 July 2003, pp. 1286-1291. doi:10.1109/AIM.2003.1225528

[6] X. Wang and G. Peng, "Modeling and Control for Pneumatic Manipulator Based on Dynamic Neural Network," Proceeding of IEEE International Conference on Systems, Man and Cybernetics, Washington, DC, 5-8 October 2003, pp. 2231-2236.

[7] G. Kothapalli and M. Y. Hassan, "Design of a Neural Network Based Intelligent PI Controller for a Pneumatic System," IAENG International Journal of Computer Science, Vol. 35, No. 2, 2008, pp. 217-225.

[8] S. Taghizadeh, B. Surgenor and M. Abu Mallouh, "Control of a Pneumatic Gantry Robot with Adaptive Neural Network Compensation," Proceeding of 34th Mechanisms and Robotics Conference, Montreal, 15-18 August 2010

[9] F. L. Lewis, "Neural Network Control of Robot Manipulators," IEEE Expert, Vol. 11, No. 3, 1996, pp. 64-75. doi:10.1109/64.506755

[10] G. Campa, M. Fravolini and M. Napolitano, "A Library of Adaptive Neural Networks for Control Purposes," 2002 IEEE International Symposium on Computer Aided Control System Design, Glasgow, 18-20 September 2002, pp. 115-120. doi:10.1109/CACSD.2002.1036939

[11] F. W. Lewis, S. Jagannathan and A. Yesildirak, "Neural Network Control of Robot Manipulators and Non-Linear Systems," Taylor \& Francis, London, 1999.

[12] R. C. Rice, "PID Tuning Guide," Rockwell Automation, Milwaukee, 2010. 